\title{
Operating cost sensitivity to required time of arrival commands to ensure separation in optimal aircraft 4D trajectories.
}

\author{
Santi Vilardaga \\ Technical University of Catalonia - Barcelona Tech, Telecom and Aerospace Engineering \\ School of Castelldefels. Esteve Terradas, 5. Castelldefels, Catalonia (Spain). \\ Xavier Prats \\ Technical University of Catalonia - Barcelona Tech, Telecom and Aerospace Engineering \\ School of Castelldefels. Esteve Terradas, 5. Castelldefels, Catalonia (Spain).
}

\begin{abstract}
Trajectory optimisation has shown good potential to reduce environmental impact in aviation. However, a recurring problem is the loss in airspace capacity that fuel optimal procedures pose, usually overcome with speed, altitude or heading advisories that lead to more costly trajectories. This paper aims at the quantification in terms of fuel and time consumption of implementing suboptimal trajectories in a 4D trajectory context that use required times of arrival at specific navigation fixes. A case study is presented by simulating conflicting Airbus A320 departures from two major airports in Catalonia. It is shown how requiring an aircraft to arrive at a waypoint early or late leads to increased fuel burn. In addition, the efficiency of such methods to resolve air traffic conflicts is studied in terms of both fuel burn and resulting aircraft separations. Finally, various scenarios are studied reflecting various airline preferences with regards to cost and fuel burn, as well as different route and conflict geometries for a broader scope of study.
\end{abstract}

Keywords: Air Traffic Management, Trajectory Optimisation, Air Traffic

\footnotetext{
Email addresses: santi.vilardaga@upc.edu (Santi Vilardaga), xavier.prats@upc.edu (Xavier Prats)
}

Preprint submitted to Elsevier

Friday $23^{\text {rd }}$ October, 2015 
Efficiency, Cost Index, Trajectory Based Operations, Conflict Resolution, Continuous Climb Departures

\section{Introduction}

One of the major drivers for research and development in the Single European Sky ATM Research programme (SESAR) and the Next Generation Air Transportation System (NextGen) programmes is the improvement of air transport efficiency in terms of economic and environmental impact (SESAR Consortium, 2012),(Federal Aviation Administration, 2014). New technologies and procedures for future air traffic management (ATM) and on-board systems and operations are being investigated and proposed. More and more, the air traffic system is seeking benefit from initiatives such as Continuous Climb Operations (CCO), Continuous Cruise Climbs (CCC), and Continuous Descent Operations (CDO), which reduce fuel in specific phases of the flight. However, such operations are hugely dependent on multiple characteristics of each aircraft (such as aircraft performance, weights or operating procedures) and meteorological conditions. This produces a great variety of individually optimal vertical and speed profiles that complicate the task of separating the traffic, ultimately impacting negatively on airspace capacity (Johnson, 2011).

Currently, separation assurance in dense terminal manoeuvring areas (TMAs) is firstly tackled at a strategic level, via complex lateral trajectory profiles and imposing altitude and velocity constraints throughout these standard procedures. Additionally, the air traffic controller operator (ATCo) will ultimately guarantee the separation at a tactical level and, if required, will issue vectoring commands (i.e. heading changes), level off aircraft at intermediate altitudes or restrict speeds at some segments. Such operations prevent the aircraft from flying optimised trajectories (i.e. CDO and $\mathrm{CCO}$ ), increasing fuel and pollutant emissions and producing a larger noise impact in the vicinity of the airport.

To prevent this negative impact in efficiency and/or airspace capacity, research has been performed on the integration of continuous operations in dense 
TMAs. An example of this is the Oceanic Tailored Arrivals program, currently in place at San Francisco airport (Coppenbarger et al. 2004). These arrivals are supported by the En-route Descent Advisor (EDA) developed by NASA-AMES, which is able to compute conflict-free optimal descent trajectories and satisfy a given arrival fix metering by issuing speed advisories to participating aircraft (Coppenbarger et al., 2007). Another example is the project Environmentally Responsible Air Transport (ERAT), which developed methods and systems to reduce environmental impact using continuous arrival and departure procedures inside high density TMAs in Europe (ERAT Consortium, 2012). Alternatively, Chaloulos et al. (2010) propose imperceptible speed adjustments to minimise the risk of potential conflicts and lessen the workload of ATCo, impacting positively into airspace capacity.

The current paradigm for air traffic management involves strict air traffic control (ATC) procedures and lacks advanced automation. Therefore, the study of $4 \mathrm{D}$ conflict-free trajectory optimisation has mostly been regarded as long term research, well away from implementation. The SESAR and NextGen programs aim to introduce Trajectory Based Operations (TBO), an enabler for trajectory optimisation implementation. For example, Soler et al. (2014a) proposes the implementation of applicable speed and heading advisories to current operations to resolve conflicts in cruise phases using TBO operational concept. Bronsvoort (2014) assesses the application of TBO air-ground synchronisation concepts with current technologies and its predictability issues. Finally, De Jong (2014) presents the application of CDO within high traffic operations with the use of time advisories, or required time of arrival (RTA) at given fixes.

Along these lines, this paper presents an optimisation framework that proposes optimal trajectories in a dense traffic area. Aiming at continuous vertical profiles while minimising the negative impact on airspace capacity, 4D optimal flight paths are computed with strict time constraints at fixes along the route. When the ATCo gives such advisories to account for other traffic, the aircraft are consequently deviated from their optimal profile. Moreover, such requests might come inflated with wider safety margins. The objective of this paper is 
twofold: firstly to present a novel framework that generates optimal trajectories with complex dynamics and constraints, and secondly to assess the impact on total cost of assigning RTAs, and their effectiveness in traffic separation. The total cost is computed with respect to the airline's cost index strategy. The described trajectory optimisation framework builds upon previous research by the authors (Vilardaga and Prats, 2014, Vilardaga et al. 2014). In it, a continuous multiphase optimal control problem formulation is created, taking into account spatial and temporal constraints.

This paper is organised as follows. Section 2 lays out the dynamic model of the aircraft and the problem formulation, including the flight phases and operational constraints. Section 3 describes the scenario that has been implemented in the case study. Section 4 presents the results divided into three separate sections: section 4.1 presents a sensitivity study of fuel to the different RTAs and its success in conflict resolution; section 4.2 presents a sensitivity study of the total cost to the different RTAs and Cost Index values, with its impact to the conflict geometry; and section 4.3 presents a sensitivity study of the total cost to the different RTAs and the distance to the RTA fix. Finally, section 5 presents the authors' conclusions.

\section{4D trajectory optimisation}

Aircraft trajectory predictability stands as an enabler for efficient and safe operations in ATM. Currently, aircraft separation is mainly handled at a strategic level by spacing air routes in order to minimise potential airborne encounters, and at a tactical level by giving open instructions to individual aircraft to remove any remaining conflict. However, this usually leads to inefficient operations. One of the main solutions to this issue is the concept of TBO, which relies on pre-established conflict-free efficient trajectories. The most common and wellaccepted implementation of this concept is based on time advisories throughout the trajectory in form of RTA at specific fixes along the route. However, the impact on fuel that these RTA have against continuous operations (CDO and 
$\mathrm{CCO}$ ) in the TMAs has not yet been assessed. The contribution of this paper is twofold: first, we present an optimisation framework that computes efficient TBO and second, we present a case study that assesses the impact in fuel of applying RTA to trajectories within a TMA.

\subsection{Literature review}

There are multiple approaches to the computation of a trajectory that complies with a set of constraints. On the one hand, trajectory prediction methods are suitable for generating quite fast and accurate solutions, depending on the complexity of the control strategy applied when integrating the equations of motion over time (PID, adaptive control, etc.) (Mondoloni, 2009). However, when dealing with highly constrained problems (complex dynamics, RTA, waypoints, etc.) this technique becomes inefficient and delivers slow and non-optimal results. On the other hand, optimisation techniques allow the definition of a cost functional that is minimised while complying with all the problem constraints. This can be approached by means of sampling-based path planning algorithms such as Dijkstra, $A^{*}, R R T^{*}$, etc (Yang et al., 2014) or stochastic optimisation methods such as Genetic Algorithms, Causal Models, etc. (Ruiz et al., 2014). However, such algorithms present a difficult trade-off between an accurate dynamics model and the computational burden of generating the solution space (samples), which can only rely on an heuristic for an informed growth. Another mathematical approach is to formulate a continuous and constrained optimal control problem. Although there are some methods to solve this problem analytically, see for instance (Franco et al., 2010, Franco and Rivas, 2014) and the references therein, these depend on big mathematical simplifications such as singular arc approximation, as well as very limited operational applications (constant altitude and speed, etc.). These limitations can be removed using a numerical approach as explained in the following paragraph.

Using optimal control, in order to cope with the important non-linearities in the different equations, and to be able to optimise multiple-phase complex aircraft trajectories, we convert the infinite-dimensional original problem into 
a finite-dimensional non-linear programming (NLP) problem with a finite set of decision variables in the time interval $\left[t_{0}, t_{f}\right]$ (Betts, 2010). This allows the definition of complex trajectories while complying with the set of dynamic equations, the given multi-phase constraints and minimising a cost functional, providing thus an accurate and flexible optimal trajectory framework.

In the literature, there are a few examples that use optimal control for trajectory optimisation in ATM. For instance, Prats et al. (2011) use a similar approach to optimise departing trajectories to reduce noise in the vicinities of an airport. Also, Soler et al. (2014b) define a mixed-integer optimal control problem for flight planning optimisation purposes. Visser and Hartjes (2014) describe a methodology for optimal flight paths between city-pairs minimising environmental footprint and airline cost criteria. Finally, Prats et al. 2015) present results on continuous descent trajectories in high density traffic with RTAs. Each of these has a similar methodology, but key aspects differ on the application of some mathematical assumptions (some use fixed altitudes, some have no lateral freedom, etc.), and the application scenario, with a different concept of operation (free-routing vs. standard procedures, ATCo restrictions, etc.).

\subsection{Problem formulation}

Let $\boldsymbol{x}(t) \in \mathbb{R}^{n_{x}}$ be the state vector describing the trajectory of the aircraft over time $t$ and $\boldsymbol{u}(t) \in \mathbb{R}^{n_{u}}$ the control vector that leads to a specific trajectory.

Our goal is to find the best trajectory that minimises the following cost functional:

$$
J=\int_{t_{0}}^{t_{f}}[F F(\boldsymbol{x}, \boldsymbol{u})+C I] \mathrm{d} t .
$$

The Cost Index $(C I)$ scalar relates the cost of time to the cost of fuel $(F F)$ and takes into account different airline policies. Moreover, the value of $t_{f}$ is a decision variable itself and will be fixed by the optimisation algorithm.

We have formulated an optimal control problem, the solution to which minimises the objective defined in Eq. (1) with the state and control vectors defined 
as follows:

$$
\begin{aligned}
& \boldsymbol{x}=\left[\begin{array}{lllllll}
v & \gamma & \chi & e & n & h & m
\end{array}\right] \\
& \boldsymbol{u}=\left[\begin{array}{llll}
n_{z} & \phi & \pi
\end{array}\right]
\end{aligned}
$$

where $n$ and $e$ represent the spatial location of the aircraft in north and east coordinates respectively, $h$ is the geometric altitude, $v$ is the true airspeed, $\gamma$ the aerodynamic flight path angle, $\chi$ the heading and $\phi$ the bank angle. Furthermore, we model the dynamics of the mass of the aircraft $m$. The load factor $\left(n_{z}\right)$ is defined as the relation between the aerodynamic lift force and the aircraft weight. Finally $\pi$ represents the throttle setting.

In order to guarantee a feasible and acceptable trajectory, as a result of this optimisation process, several constraints must be considered. In particular, the dynamics of the system (dynamics of the state vector), expressed by non-linear differential equations. Furthermore, additional algebraic constraints either at the initial/final points or all along the trajectory must be specified. The next subsections describe the mathematical formulation of these constraints.

\subsubsection{Aircraft dynamics}

In this paper, a point-mass representation of the aircraft is used, where forces apply at its centre of gravity. A situation with benign and constant winds in a flat non-rotating earth has been assumed. The equations of motion are written as follows (Prats, 2010): 


$$
\begin{aligned}
& \frac{\mathrm{d} v}{\mathrm{~d} t}=\dot{v}=\frac{1}{m}(T-D-m g \sin \gamma) \\
& \frac{\mathrm{d} \gamma}{\mathrm{d} t}=\dot{\gamma}=\frac{g}{v}\left(n_{z} \cos \phi-\cos \gamma\right) \\
& \frac{\mathrm{d} \chi}{\mathrm{d} t}=\dot{\chi}=\frac{g}{\sin \phi} n_{z} \\
& \frac{\mathrm{d} e}{\mathrm{~d} t}=\dot{e} \gamma=v \cos \gamma \sin \chi+W_{e} \\
& \frac{\mathrm{d} n}{\mathrm{~d} t}=\dot{n}=v \cos \gamma \cos \chi+W_{n} \\
& \frac{\mathrm{d} h}{\mathrm{~d} t}=\dot{h}=v \sin \gamma \\
& \frac{\mathrm{d} m}{\mathrm{~d} t}=\dot{m}=-F F
\end{aligned}
$$

where $T$ is the aircraft thrust, $D$ is the aerodynamic drag, $W_{n}$ and $W_{e}$ are the north and east wind components respectively and $g$ is the gravity acceleration.

Regarding the atmosphere, a set of polynomial approximations of weather forecast coming from GRIB ${ }^{1}$ files has been implemented to define the density $\rho$, pressure $p$ and temperature $\tau$ magnitude as functions of the altitude and geographic location.

All aerodynamic and engine parameters are represented by continuous polynomials, that ensure continuity for the first and second derivatives as it is required for the numerical solvers used here. Aerodynamic Lift $(L)$ and Drag $(D)$ forces are modelled considering air compressibility effects, which cannot be neglected for nominal cruising speeds of typical commercial aircraft (between M.78 and M.82 approximately). Tabulated aircraft aerodynamic data has been obtained from Airbus PEP software suite, which provided us with accurate (and certified) values for aerodynamic Drag and engine performance for different flight conditions. More details about the specific implementation are found in Vilardaga and Prats (2014).

\footnotetext{
${ }^{1}$ General Regularly-distributed Information in Binary form: common format for storing and sharing weather data.
} 


\subsubsection{Operational constraints}

Besides the equations of motion described above, the problem is further constrained by additional equations that take into account several operational restrictions. Since the operating speeds are always expressed in calibrated air-

speed $\left(v_{C A S}\right)$, an extra constraint equation to relate this speed to the true airspeed $(v)$ is added:

$$
v_{C A S}=\sqrt{\frac{2 p_{0}}{\mu \rho_{0}}\left[\left(\delta\left(\left(\frac{\mu v^{2}}{2 R \tau}+1\right)^{\frac{1}{\mu}}-1\right)+1\right)^{\mu}-1\right]}
$$

where $\mu=\frac{\gamma_{a}-1}{\gamma_{a}}, \gamma_{a}$ is the specific heat ratio of the air and $R$ the perfect gas constant.

Some other constraints are specified as a function of the along path distance $(s)$, which although it is not a state variable its dynamics are modelled as:

$$
\frac{\mathrm{d} s}{\mathrm{~d} t}=\dot{s}=\sqrt{\dot{e}^{2}+\dot{n}^{2}}
$$

Table 1 depicts the constraints considered in the optimisation problem. Many of these are operational constraints, either to stay within the flight envelope (for instance, $V_{M C A}$ for the minimum aircraft control speed in the air and $V_{M O}$ for the maximum aircraft operating speed) or comply with ATM constraints such as CAS profiles and ground obstacle avoidance. Additionally, bounding constraints on $n_{z}$ and $\phi$ are defined following civil aviation standards. More information on optimal control formulation and resolution techniques used in this research can be found in Betts and Cramer (1995).

Furthermore, specific constraints can be set for the state variables at specific nodes to represent the initial and final state of the optimisation problem as well as intermediate restrictions (such as waypoints, RTAs, etc.).

Finally, some collocation and link equations ensure that the different state nodes (collocation points) within a phase are correctly linked to the previous phases in compliance with the dynamic models, and that the phases relate to the time continuum they represent as explained in the following subsection. 
Table 1: Constraints in the optimal control problem

\begin{tabular}{cc}
\hline Constraint & Definition \\
\hline Operating airspeeds & $V_{M C A} \leq v_{C A S}(t) \leq V_{M O}$ \\
No deceleration allowed & $\dot{v}_{C A S}(t) \geq 0$ \\
No descent allowed & $\dot{h}(t) \geq 0$ \\
Minimum climb gradient & $h(t) \geq 0.033 s(t)$ \\
Load factor & $0.85 \leq n_{z}(t) \leq 1.15$ \\
Bank angle & $-25^{\circ} \leq \phi(t) \leq 25^{\circ}$ \\
\hline
\end{tabular}

\subsubsection{Multiphase modelling}

Aircraft trajectories are divided into multiple flight phases with specific performance values assigned to each phase. During the first phase of its trajectory, an aircraft will be at maximum take-off thrust climbing without the possibility of turning or making changes to the aerodynamic configuration. In many studies, this phase is not contemplated given the low degrees of freedom that pose these operational constraints. The phases following this initial climb phase are defined by the different and sequential aerodynamic changes (flaps/slats retraction). These aerodynamic configuration changes are typically executed at predefined speed steps.

Besides, the departing aircraft follows a route specified by a set of vertical, lateral and speed constraints described in the published Standard Instrument Procedures (SID). Additionally, such constraints may also be issued by an ATCo at a tactical level, along with one or several RTAs in a $4 \mathrm{D}$ trajectory scenario.

In the previous paragraphs we have specified two disconnected types of phases: those related to aerodynamic configuration of the aircraft (e.g. changes in flaps, gear, etc.) and those related to ATM procedures (changes in heading, altitudes, etc.). In other words, the order of which operational events occur is independent to the order of which ATM events occur. For example, a geographical fix on the procedure could come before or after the moment the aircraft 
transitions from one aerodynamic configuration to the following one. Even for the same aircraft type, depending on the weather conditions and the mass of the aircraft the order of these events will differ and it is impossible to know beforehand. Due to the nature of optimal control problems, the unordered use of phases becomes a challenge to be solved.

A possible solution to overcome this issue is to reformulate the optimisation problem into a mixed-integer non-linear programming problem and use integer decision variables to find the optimal sequence of ATM and operational phases. A fine example of this approach for aircraft trajectory optimisation is given by Bonami et al. (2013). This method, however, increases the complexity of the problem and might negatively impact the computational time to obtain a solution. Another solution is to use continuous and twice-differentiable switching functions to model certain phase changes. This method has the negative sideeffect that it adds non-linearities to the model (greater computational times and possible convergence difficulties), and the minor drawback of having a transition effect around the switching value. Both issues are directly related, since a less steep function at the switching point (and thus smoother for the NLP Solver) results in a bigger transition effect, and vice versa. Hence, a trade-off must be sought (Hartjes et al. 2010$)$.

In this paper, we have used these switching functions to take into consideration the changes in aerodynamic configurations, which allows the use of optimal control phases to model the ordered list of fixes defining the lateral and vertical route. With this solution, we are able to compute a full trajectory from a set of initial conditions to a set of final conditions, including one or more RTAs in the fixes along the route using a constrained multi-phase optimal control problem.

\subsubsection{Fixed lateral route}

In the current concept of operations, standard navigation procedures are in place in such a way that they minimise the number of conflicts at a strategic level. Yet, in busy TMAs conflicts may still appear and it is very common that ATCo act tactically by deviating aircraft from their nominal route giving direct 
heading instructions (the so called radar vectors) and/or levelling-off the aircraft to ensure vertical separation at the conflict point. Yet, future paradigms like those described in SESAR and NextGen programmes tend to keep the lateral trajectory as close as possible to the nominal trajectory initially planned by the aircraft operator. For this reason, in this paper we fix the lateral path of aircraft and we analyse the use of RTAs to ensure a given separation between conflicting aircraft.

Because a trajectory can be a very complex curve, we rely on polynomial fitting to model a predicted reference trajectory (Gong and Sadovsky, 2010). To this end, we find the fuel-optimal trajectory of each aircraft, which is then approximated with curves represented by basis splines (B-splines). A cubic B-spline is a continuous twice-differentiable function represented by piecewise polynomials of order three. As opposed to higher degree polynomials, these provide an accurate fitting and have been demonstrated to perform well with NLP optimisation (Betts and Cramer, 1995, Vilardaga et al. 2014) as they can be very smooth.

Effectively, through many different simulations, this solution has been very reliable and robust, showing good performance. Our problem creates two splines that represent the ownship's north and east coordinates over the along path distance $(s)$. At the optimisation process, the solver iteratively calls these curves that represent the geographical position, and the $n$ and $e$ variables in the state vector are constrained following these over $s$ :

$$
\begin{aligned}
n(t) & =\Gamma_{n}(s(t)) \\
e(t) & =\Gamma_{e}(s(t))
\end{aligned}
$$

where $\Gamma_{n}$ and $\Gamma_{e}$ are the splines modelling the (fixed) lateral route of the aircraft. The Root Mean Square (RMS) of the error between the resulting polynomial approximation and the original trajectory is negligible. 


\subsection{Optimisation framework}

The optimisation framework described in this paper integrates different modules. The software tool has been mainly developed in $\mathrm{C}++$ and General Algebraic modelling System (GAMS ${ }^{2}$ ). The core part of the optimiser is written in GAMS, given the facility and robustness it provides to implement optimal control problems and the multiple NLP solver engines to which it seamlessly links. All other software modules are written in $\mathrm{C}++$, including a wrapper to the core functionality. Thus, we can dynamically define and load scenarios (described by a Flight Plan and many other problem parameters), prepare them for the optimisation and gather the results once done. The flexibility of the described framework allows for an easy implementation of different flight profiles, from completely unconstrained continuous operations, to defined standard procedures such as Noise Abatement Departure Procedures (NADP), standard instrument departures and arrivals (SID, STAR), etc. as well as any ATC constraint.

\section{Case study}

A typical conflict that is encountered within a TMA is usually due to the close proximity of two airports (like it happens for instance in the Bay Area around San Francisco airport or in the New York Area, and mostly all over Europe) or between departures and arrivals in the same airport. For the purpose of this paper, we have prepared a scenario following close-to-real life operations in two major airports in the Catalonia region. The Airbus A320, a typical twin-engine narrow-body aircraft, has been simulated in all the cases explained below. Aircraft departing Barcelona (LEBL) and Reus (LERS) airports have been considered and we have focused in two simultaneous departures from each airport creating an eventual conflict. In Reus, the current eastwards departure procedure BCN1S makes a long detour to strategically deconflict departing traf-

\footnotetext{
${ }^{2}$ http://www.gams.com
} 
fic with inbound and outbound traffic of Barcelona airport (see Figure 1). In SESAR and NextGen, with the concept of Trajectory Based Operations (TBO), these long detours may be replaced by pre-cleared 4D trajectories. Hence, in this paper we study a new, more fuel efficient, straight departure route as depicted in the same figure.

Let us call by RES the trajectory of an aircraft departing from Reus and following this new departure and by $\mathrm{BCN}$, the trajectory of another aircraft that departs Barcelona to the west through the GRAUS3W SID. Figure 1 shows the lateral routes as resulting from the optimiser, overlaid on the charts defined in the Spanish Aeronautical Information Publications (AIP) Aeropuertos Españoles y Navegación Aérea, 2012, 2010).

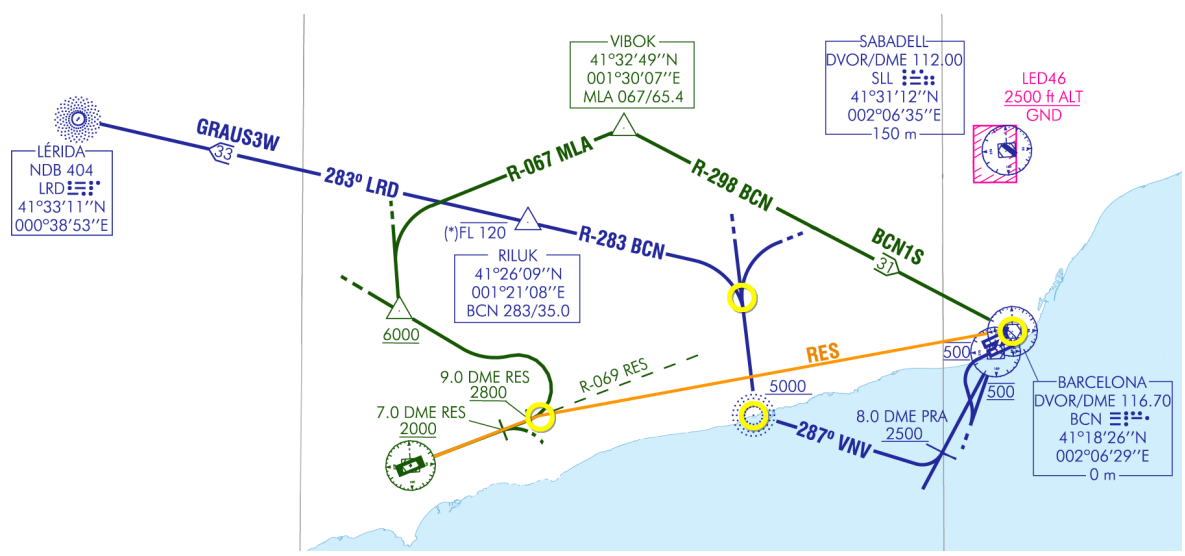

Figure 1: Published standard instrument departures for Barcelona (GRAUS3W, blue) and Reus (BCN1S, green) and proposed direct route (RES, orange).

This new direct route from Reus is more likely to create conflicts with traffic in Barcelona and effectively, two aircraft departing at approximately the same time with a similar take-off mass enter in conflict soon after take-off. Therefore, some action is required to prevent the loss of separation. The following paragraphs describe potential actions broken into different cases. RES and BCN trajectories are simulated through all cases with the same ending condition at cruise altitude of FL320 and final speed of Mach 0.73 . 


\section{Case A: Baseline}

In this case a cost-optimal baseline scenario has been defined from the ground to cruise altitude without traffic related constraints. Effectively, these optimal trajectories cannot be flown due to a loss of separation with the other aircraft, but they lay out the resulting conflict geometry and this case is also used as the cost-optimal reference trajectory for comparison with all other cases.

\section{Case B: RTA at a fix}

Following 4D Trajectory Based Operations concepts, the strategy to keep lateral separation between aircraft could be to assign a requested time of arrival at a certain fix along each aircraft's route so they reach the conflicting area at an earlier or later time, thus preventing the conflict. The exact time deviation that is needed is unknown and will vary for every scenario under study. This is why, in order to fulfil the objective of this paper, we have defined a set of feasible earlier and later RTAs, aiming at the quantification of the impact in fuel that such non-optimal time requests pose to the aircraft. These set of simulations have a fixed lateral route as described in section 2.2.4. Therefore, requesting faster or slower trajectories will only have an impact on the vertical and speed profiles of the aircraft. The fix to which the RTA is requested depends on the geometry of the conflict and is explained below.

\section{Case C: Level-off segment}

Current ATC procedures tend to resolve this type of situation by issuing directives to at least one of the involved aircraft, typically resulting in a level-off flight segment. The duration of this segment depends on the TMA complexity and ATC practices. In this paper a level segment of 
$18 \mathrm{NM}$ has been simulated, which was found to be the average segment length in a study in New York and Paris TMAs (Thompson et al., 2013).

\section{Case D: Collaborative conflict-free optimisation}

Finally, case D includes both aircraft in a single optimisation problem with separation assurance. This gives the overall fuel-optimal trajectories that both aircraft could follow without violating the separation constraints.

With the optimisation framework described in the previous section, cases A to $\mathrm{D}$ are studied as follows. First, we find out at what latitude and longitude the trajectories enter in conflict along their fuel-optimal trajectories (case A). From this geographical location we then select the adequate fix in each aircraft's flight plan where the RTA will be requested (see yellow circles in figure 1 for proposed fixes in the example). For earlier RTAs, the selected fix will be the one that comes after the geographical encounter (we want to clear as fast as possible the conflicting leg). For later RTAs it will be the one before (we want to arrive later at the conflicting leg).

We then iteratively assign earlier and later times to the corresponding fix, starting at the optimal time of overfly up to the earliest and latest feasible RTA respectively (case B). Initial and final conditions (including speed) are kept equal for all cases. Each new trajectory gives different fuel consumption, and modifies the conflict geometry, possibly completely removing it. Finally we compare it to current operations assuming a level-off segment (case C) and to potential future operations in a collaborative conflict-free optimisation (case D).

In an effort to adapt the optimiser to current climb operations, the CAS profile is restricted through the climb procedure as shown in table 1 . In short, CAS decelerations are not allowed. However, earlier RTAs will most probably need higher speeds to meet the RTA and will not be able to comply with the final speed constraint at the ending point. To make this possible, we introduce a deceleration phase (at constant $\dot{v}_{C A S}$ ) that only happens immediately after 
the RTA is met to allow the aircraft to slow down to the desired speed. This deceleration phase is only allowed in earlier RTAs.

\section{Numerical results}

This section lays out the numerical results for the different cases (A to D) described in section 3 First of all, we study the impact of RTAs applied individually to one trajectory, or in combination between two trajectories. At the end, two sensitivity studies are presented: one towards cost index and another one towards distance of encounter of the RTA fix.

\subsection{Numerical results for $C I=0 \mathrm{~kg} / \mathrm{min}$}

This section shows numerical results involving comparisons for all cases when $C I=0 \mathrm{~kg} / \mathrm{min}$ (i.e. only fuel is minimised). First, Case A trajectories are computed (as displayed in Figure 1), where the aircraft fly fuel-optimal trajectories for both departures regardless of the potential conflict at the crossing point. Then, the sensitivity study for Case B is studied individually (only one aircraft receives an RTA, section 4.1.1) and in combination (both aircraft receive an RTA, section 4.1.2 , and is compared with cases C and D.

\subsubsection{Individual RTAs}

The results for case B are summarised in figure 2, showing the differences in fuel and time with respect to the baseline scenario (Case A) for different RTAs. It should be noted that $\triangle \mathrm{RTA}=0 \mathrm{~s}$ means no time deviation from the original optimal trajectory (Case A). Furthermore, we have analysed two possible situations: $\mathrm{BCN}$ is the ownship and must change the trajectory according to the new RTA and vice-versa. In both cases, the intruder is always flying its corresponding optimal trajectory of Case A.

Given the different geometries in BCN and RES trajectories, the RTAs of study are not the same. Fore example, BCN has $33 \mathrm{NM}$ of along track distance before reaching the fix where earlier RTAs are applied, whereas RES has a longer stretch: 43 NM. This explains why the magnitude of the earliest feasible $\Delta$ RTA 


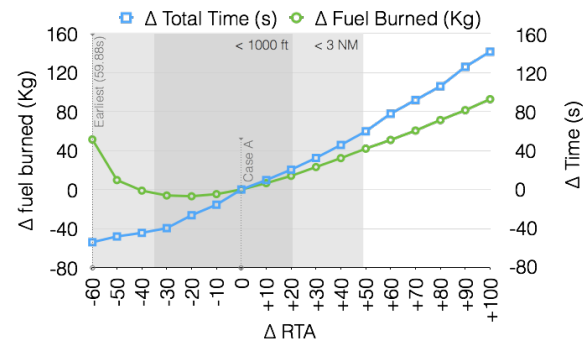

(a) Ownship: BCN, Intruder: RES.

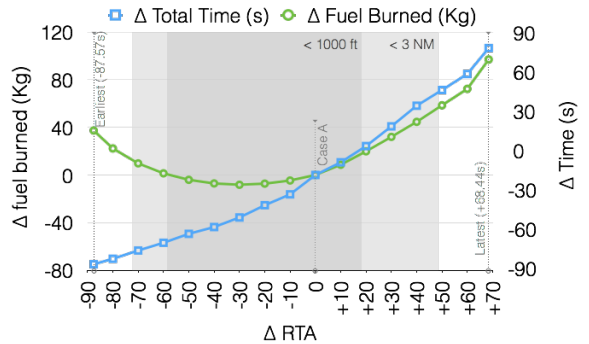

(b) Ownship: RES, Intruder: BCN.

Figure 2: Increase in fuel burned and trajectory time evolution for different RTAs in case B.

for $\mathrm{BCN}$ is lower than for RES, which has a longer stretch to gain speed. For later $\triangle \mathrm{RTA}$ things are inverted, with BCN having the possibility of reaching the fix where the later RTAs are applied at $\Delta \mathrm{RTA}=+204 \mathrm{~s}$ whereas RES can only go as far as $\Delta \mathrm{RTA}=+68 \mathrm{~s}$, mainly because the latter has a shorter leg to generate the delay (specifically, BCN has $23 \mathrm{NM}$ and RES $9 \mathrm{NM}$ ).

The expected conclusion is that in general earlier and later RTAs are less efficient than the optimal reference for both depicted trajectories. However, for some earlier $\triangle R T A$ this is not the case. The reasons for that are found in the introduction of the deceleration phase after the metering fix, as described at the end of section 3 . Without this phase, calibrated airspeed (CAS) decelerations are not allowed, which indirectly restricts the vertical profile (for the same true airspeed (TAS), CAS decreases as the altitude increases). However, from the optimiser's perspective, it is more fuel-efficient to climb at a higher speed with a deceleration phase at the end (see figure 3). For this reason, the optimiser reaches lower fuel consumptions than the more restrictive optimal reference. Other solutions would involve restrictions in TAS instead of CAS (less restrictive in the altitude profile), but this poses a big shift in air operations and has been deliberately kept out of the scope of this paper. Still, acknowledging this aspect, the results in fuel are perfectly valid for the proposed sensitivity analysis.

As previously explained, the baseline case A does not keep the required horizontal and vertical separation of $3 \mathrm{NM}$ and $1000 \mathrm{ft}$, respectively, between the 


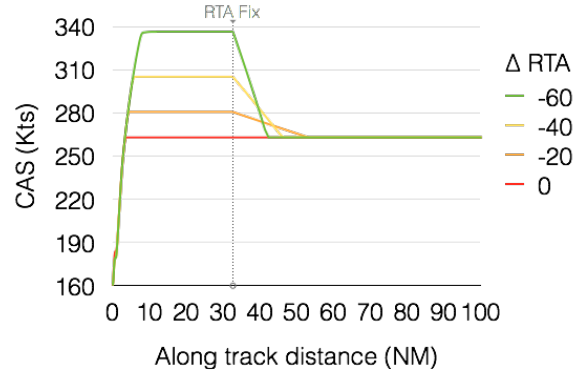

(a) Ownship: BCN

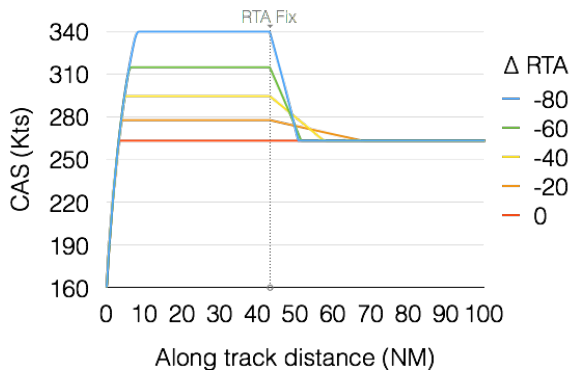

(b) Ownship: RES

Figure 3: CAS profile for some earlier RTAs in BCN and RES trajectories.

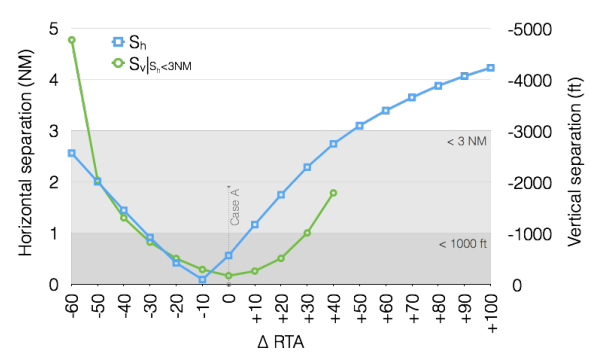

(a) Ownship: BCN, Intruder: RES.

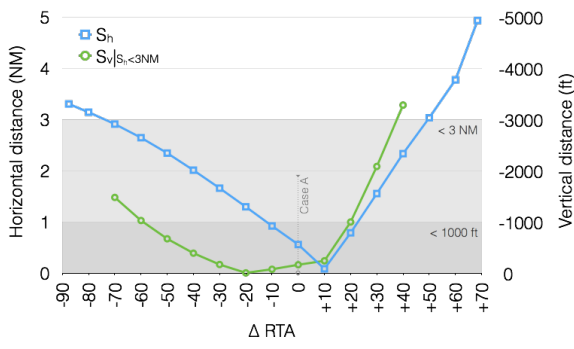

(b) Ownship: RES, Intruder: BCN.

Figure 4: Minimum aircraft separation between ownship and intruder for different RTAs in case B with $C I=0 \mathrm{~kg} / \mathrm{min}$.

involved aircraft. In figure 2 we have presented a sensitivity study of the impact of different RTA to the fuel consumption. But how do these RTA perform at keeping the required separation between aircraft? Figure 4 shows the minimum aircraft horizontal separation $\left(S_{h}\right)$ for each RTA of study. Additionally, the minimum vertical separation when the horizontal separation is not granted (i.e. less than $3 \mathrm{NM}$ ) is defined as $\left.S_{v}\right|_{S_{h}<3 \mathrm{NM}}$.

As an example, if BCN aircraft would reach the conflicting leg 50 seconds later than its fuel-optimal time, it would completely remove the conflict by keeping the required minimum horizontal separation with RES throughout the flight. Contrasted with figure 2, this represents a $41.9 \mathrm{~kg}$ increase in fuel, reaching the final point $60 \mathrm{~s}$ later. Similarly, RES could itself prevent the loss of horizontal 
separation by reaching the area $80 \mathrm{~s}$ earlier, with a fuel increase of over $22.3 \mathrm{~kg}$, gaining $70 \mathrm{~s}$. However, from figure 4 , it is clearly visible that the vertical separation is always achieved before the horizontal. Effectively, the conflict could be removed with the use of RTAs (e.g. RES+20, RES-60, $\mathrm{BCN}+30$ or $\mathrm{BCN}-40$ ) with fuel increases ranging from $-1 \mathrm{~kg}$ to $23 \mathrm{~kg}$. The small impact in fuel can be explained because both aircraft are climbing and a slight early vertical deviation will keep the $1000 \mathrm{ft}$ separation without further efforts. In comparison, a level-off phase imposed to one of the aircraft (case C) would represent $47.6 \mathrm{~kg}$ more, with a total flight duration 68 seconds longer than the fuel-optimal path (RES) or $50 \mathrm{~kg}$ and $73 \mathrm{~s}(\mathrm{BCN})$. Some of these figures are summarised in table 2 .

Table 2: Summary of costs for some representative examples of cases A, B and C

\begin{tabular}{lcrrcc}
\hline Case & Description & $\Delta$ Fuel $(\mathrm{kg})$ & $\Delta$ Time $(\mathrm{s})$ & $S_{h}$ & $\left.S_{v}\right|_{S_{h}<3 \mathrm{NM}}$ \\
\hline Case A & Optimal Reference & 0.00 & 0.00 & 0.56 & 154 \\
Case B.1 & $\Delta R T A_{B C N}=+50 \mathrm{~s}$ & +41.9 & +60 & 3.09 & - \\
Case B.2 & $\Delta R T A_{R E S}=-80 \mathrm{~s}$ & +22.3 & -70 & 3.14 & - \\
Case B.3 & $\Delta R T A_{B C N}=+30 \mathrm{~s}$ & +23 & +32 & 2.28 & 1000 \\
Case B.4 & $\Delta R T A_{R E S}=+20 \mathrm{~s}$ & +19.8 & +24 & 0.79 & 1000 \\
Case C.1 & Level-off phase BCN & +47.6 & +68 & 0.88 & 4028 \\
Case C.2 & Level-off phase RES & +50 & +73 & 0.38 & 3830 \\
\hline
\end{tabular}

\subsubsection{Combined RTAs}

Previous results are based on issuing time constraints to only one of the involved aircraft. The study to apply an RTA to both aircraft would most surely require smaller $\Delta$ RTAs, with a presumably lower increase in fuel overall. Effectively, this study is depicted in figure 5 where different RTAs have been given to both trajectories and the minimum horizontal separation between aircraft is depicted. As for the previous results, this study calculates the fuel-optimal 


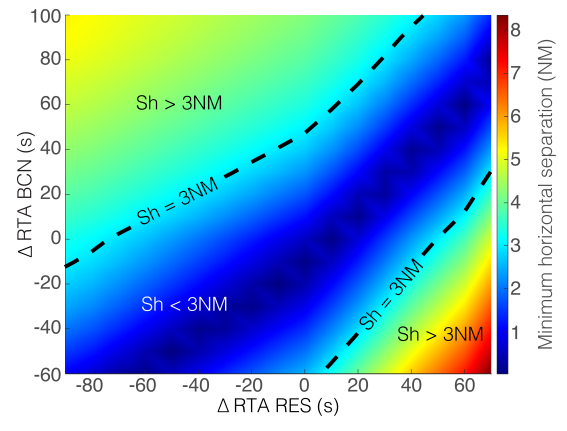

(a) Minimum horizontal separation $\left(S_{h}\right)$.

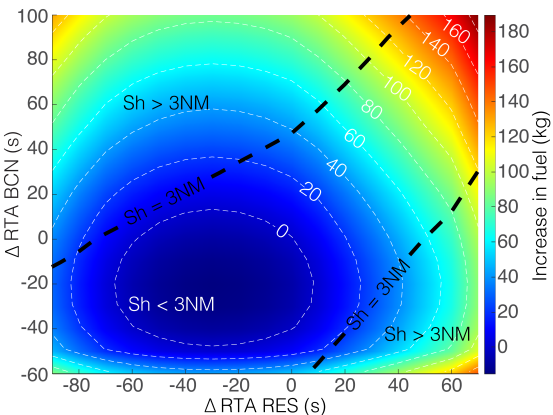

(b) Accumulated increase in fuel.

Figure 5: Effectiveness and impact in fuel of different RTAs for both trajectories in case B and $C I=0 \mathrm{~kg} / \mathrm{min}$.

trajectories for both routes, which constitute the baseline $\Delta \mathrm{RTA}_{\mathrm{BCN}}=0 \mathrm{~s}$ and $\Delta \mathrm{RTA}_{\mathrm{RES}}=0 \mathrm{~s}$, and then recalculates the trajectories iteratively incrementing and decrementing the reference by $10 \mathrm{~s}$. Compared to the previous results, this figure shows, for example, how delaying the RTA to BCN by about 30 seconds, and at the same time advancing the same amount to RES, the required horizontal separation would be granted, only implying an overall increase in fuel of $14.7 \mathrm{~kg}$, much less than the results showed above. Similarly, BCN $\Delta \mathrm{RTA}=+20 \mathrm{~s}$ and RES $\Delta \mathrm{RTA}=-50 \mathrm{~s}$ would result in $\Delta \mathrm{Fuel}=9.9 \mathrm{~kg}$. Other successful cases can be drawn from figure 5 It presents, for the given situation and predicted trajectories, the best conflict resolution using RTAs for horizontal separation. However, these results raise the need for better predictability in aircraft operations (Bronsvoort, 2014), since the predictions are used to assign the RTAs for separation. In such situation, the RTA could also be designed for vertical separation, giving thus even more efficient conflict resolution results.

Finally, the best way to find an optimal resolution for the conflict would need full collaboration with unlimited information sharing within airspace users. In such case both trajectories could be generated in a single optimisation problem that would seek the global optimum without violating the separation constraints (case D). We understand that the operational implementation of this fully col- 


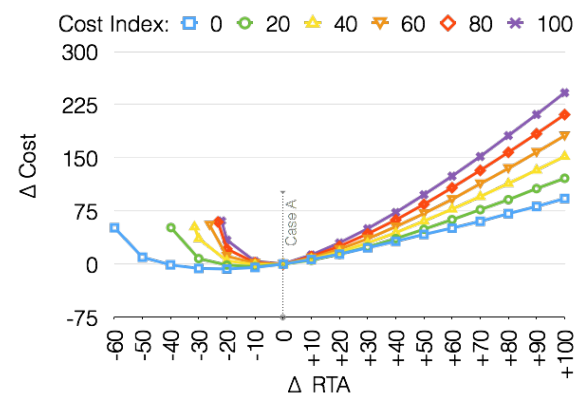

(a) Total Cost

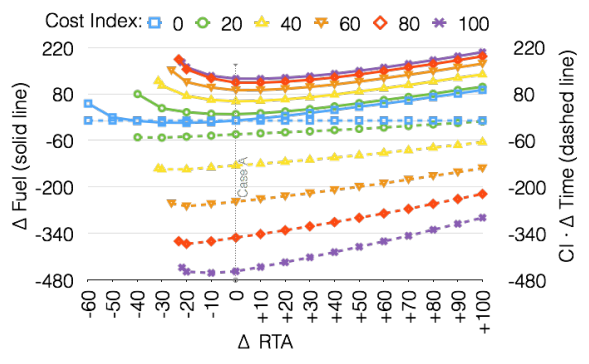

(b) Fuel and Time

Figure 6: Evolution of total cost, fuel and time for different $C I$ and different RTAs in case B (Ownship: BCN).

laborative paradigm is very complex and still far off in the future. Nevertheless, the results are very promising, giving a negligible increase in fuel when compared to the fuel-optimal baseline. This can be explained because in the example both aircraft are climbing, and small deviations in the vertical profile at the beginning do not affect too much the global consumption, but are enough to keep the vertical separation throughout the flight.

\subsection{Sensitivity study to cost index}

To this point, the results shown are based assuming the airlines are looking for the fuel-optimal trajectory (i.e. $C I=0 \mathrm{~kg} / \mathrm{min}$ ). Currently, however, this is seldom the case, due to fluctuations in fuel cost and its relation to other airline costs (mainly related to staff), as well as constraints in time. In order to broaden the scope of the current work, this section extends the previous sections' results to account for multiple $C I$ values.

Figure 6 repeats the results shown for case $\mathrm{B}$ with different example $C I$ values ranging from $C I=0 \mathrm{~kg} / \mathrm{min}$ (fuel only) to $C I=100 \mathrm{~kg} / \mathrm{min}$. For each $C I$ value, a new baseline trajectory (case A) is generated. Then, the $\triangle$ RTAs in figure 6 are with respect to each $C I$ reference optimal.

Figure 6(a) shows the cost evolution with respect to each $C I$ reference optimal. Not surprisingly, figure 6 shows how for bigger $C I$ values, later RTAs become more and more costly. However, for earlier RTAs the situation is similar: 
for a same $\Delta \mathrm{RTA}$, for higher $C I$ values, higher costs are seen. This means that even if the time dimension is more and more important in the cost function, the gains in time that faster trajectories render are hindered by the increase in fuel to achieve such trajectories. Besides, for higher $C I$ values, the baseline case $\mathrm{A}$ is in itself faster when compared to lower $C I$. Hence, advancing the RTA demands, for higher $C I$, great amounts of added fuel, for not as big a time gain.

This can be better seen in figure 6(b), where a breakdown of the cost function (Eq. 1) is presented. It shows the evolution of fuel (solid line) and CI - Time (dashed line) of each new trajectory with respect to the fuel-optimal reference (Case A). It shows how, even if the dashed line (time dimension) decreases, the solid line (fuel dimension) increases as much, or even more, for many of the cases in earlier RTAs. It is also worth noting that for $C I=80 \mathrm{~kg} / \mathrm{min}$ and $C I=100 \mathrm{~kg} / \mathrm{min}$, the results are very similar. This is due to the fact that in both cases, trajectories are approaching maximum speed values. Furthermore, this demands very low vertical speeds, so that reaching the required cruise altitude for the available along path distance is much more difficult.

The different $C I$ values also have an impact on the separation between the ownship and the intruder. In order to keep this paper to an assumable number of pages and figures, but without loss of generality, we only vary the $C I$ of the ownship (BCN, in the example) while we assume the intruder (RES) has $C I=0 \mathrm{~kg} / \mathrm{min}$. Figure 7 presents an example.

The minimum horizontal separation between BCN and RES is similar for the different $C I$ with a small shift in the time dimension. This is to be expected, since bigger $C I$ values fly faster, therefore the required $\triangle \mathrm{RTA}$ to reach the fix at a specified time instant grows. As expected, the required horizontal separation cannot be granted for earlier RTAs regardless of the $C I$ value (the earliest feasible time is the same), and a bigger $\Delta$ RTA will be needed to keep separation with a later RTA $(\triangle \mathrm{RTA}$ is computed with respect to each $C I$ optimal baseline, which is faster). More interesting is the fact that for a $C I$ greater than $60 \mathrm{~kg} / \mathrm{min}$, the vertical separation in case A is already granted. The reason for that is that higher $C I$ values have higher speeds, which result in shallower 


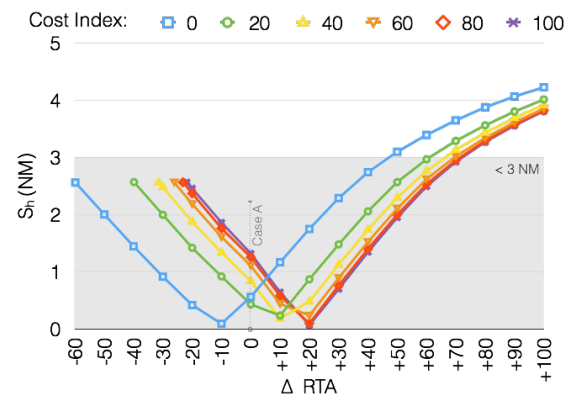

(a) Horizontal Separation $\left(S_{h}\right)$

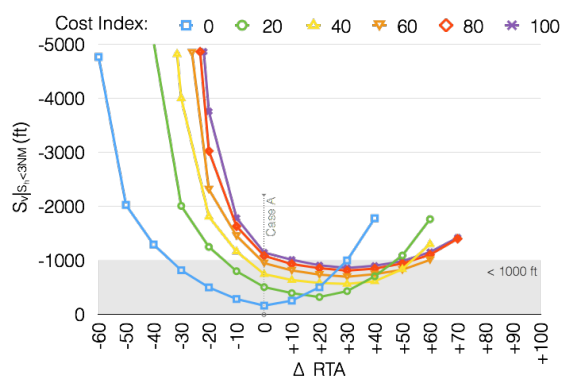

(b) Vertical Separation $\left(\left.S_{v}\right|_{S_{h}<3 \mathrm{NM}}\right)$

Figure 7: Minimum aircraft separation between ownship and intruder for different RTAs in case B with different $C I$ (Ownship: BCN, Intruder: RES with $C I=0 \mathrm{~kg} / \mathrm{min}$ ).

vertical profiles.

At the end, we can conclude that as the $C I$ value increases, the resulting cost to imposed $\triangle$ RTAs is higher. However, the different values of $C I$ have a big impact on the conflict geometry, and therefore when estimating the possibility of a loss of separation, it is very important to know the airline strategy, or at least to propose different solutions to the conflict so the airlines can take part in the decision making.

\subsection{Sensitivity study to the distance of the RTA fix}

In the previous sections we have based all the results on two trajectories departing Barcelona and Reus airports. To complement this study, it would be interesting to provide results for a larger set of scenarios, including other routes, other conflict geometries, etc. Given the fact that such study would require extensive data sets, and even so, it would be impossible to cover all possible situations, we have created a generic study that provides results that can be extrapolated to a big range of scenarios. This study is based on the assumption that the most important cause of fuel increase for a given RTA is related to the distance that the aircraft can use to comply with the time constraint.

The study is defined by one departing aircraft in a straight climbing trajectory that is requested an RTA at a fix, located at a specific along track distance 
from the runway. Figure 8 presents results for all feasible RTAs at intervals of $10 \mathrm{~s}$, for different locations of the RTA fix (from $10 \mathrm{NM}$ to $100 \mathrm{NM}$, every $10 \mathrm{NM}$ ). In it, the white space represents RTAs that are not achievable given the operational restrictions. As expected, we observe that for shorter distances there is a smaller number of feasible trajectories. Also, the increase in fuel for negative $\Delta$ RTA grows at higher rates than for positive $\Delta$ RTA. This is also true for shorter distances when compared to longer distances.

Regardless of the lateral route defined by the SID, the impact on fuel will mainly depend on the distance between the moment that the RTA is issued and the RTA fix. In the case where a departing aircraft would encounter more than one conflict throughout the climbing phase, each conflict would be resolved by the adequate RTA, and would hence be treated as an individual problem. Therefore, the results presented in this section are applicable to a wide range of scenarios and conflict geometries.

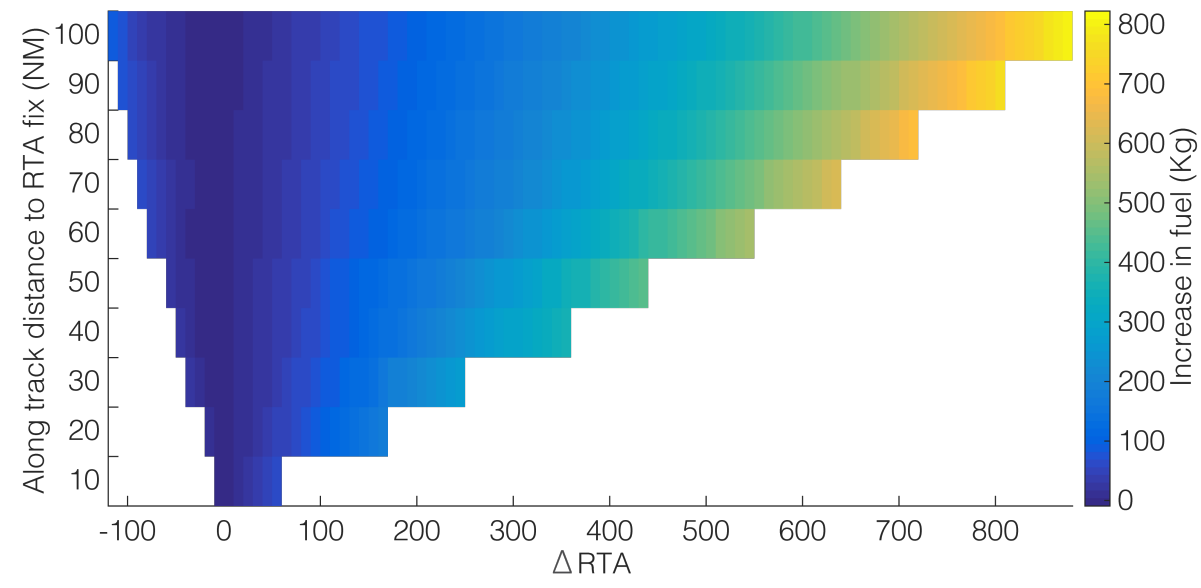

Figure 8: Sensitivity study of fuel to the distance of the RTA fix.

\section{Conclusion and further work}

In this paper we have described a framework for optimising 4D trajectories in a lateral, vertical and speed profile perspective, while meeting imposed time 
constraints for airspace capacity and aircraft separation objectives. A sensitivity study has been developed that includes many cases and RTAs to assess the impact in fuel that sub-optimal trajectories pose. As expected, RTA produce higher costs when compared to unrestricted continuous operations. However, when compared to imposing a level-off phase (as current ATCo may impose for tactical separation), the gains are considerable, reaching very low increases in cost when the RTAs are defined in collaboration to all aircraft involved in a potential conflict. Moreover, results show that vertical separation is easier to achieve than horizontal separation. Also, as a side effect, an interesting result shows how adding a deceleration phase at the climbing procedure might cause lower fuel consumption. Even if this would not be foreseen in current climb operations, it should be further quantified to assess its benefits.

Finally, a fully collaborative concept of operations has been assumed that resolves the conflict in cooperation among the involved aircraft. In the presented example, this centralised optimisation provides conflict-free trajectories with a negligible increase in cost. However, this scenario is still considered as utopic for its difficulty in operational implementation. The main complexity resides in the need for high accuracy TBO and self-separation concepts (to account for predictability errors), as well as the current lack of information sharing between airspace users.

We have presented a specific scenario where this framework could work as assistance to decision making for separation assurance with minimum cost impact using RTAs. The granularity of the different RTAs and $C I$ of study is small enough so the ATCo could easily quantify at each moment the impact in fuel of the available conflict resolution strategies and the required security buffers to account for uncertainty. However, such results are based on performance data that is, currently, not shared between airspace users (e.g. aircraft mass, cost index, etc.). Furthermore, weather predictions and updates should be taken into account for better accuracy. Therefore, there is still a need for further research in air traffic predictability in order to make this concept operational.

In a highly automated air traffic system, the framework described in this 
paper enhances the situational awareness of the ATCo by providing information of the ownship energy state in accordance to the separation with the surrounding traffic and the possibilities for efficient separation assurance.

\section{Acknowledgments}

The authors would like to thank Airbus Industrie for the use of PEP (Performance Engineers Program) suite, which allowed us to undertake realistic aircraft performance simulations. This work is partially sponsored by SESAR WP-E HALA! Research Network.

\section{References}

Aeropuertos Españoles y Navegación Aérea, 2010. Normalised Departure Chart. Standard Instrumental Procedure for Reus. RWY07.

Aeropuertos Españoles y Navegación Aérea, 2012. Normalised Departure Chart. Standard Instrumental Procedure for Barcelona / El Prat. RWY25L / RWY20.

Betts, J. T., 2010. Practical methods for optimal control and estimation using nonlinear programming, 2nd Edition. No. 19 in Advances in design and control. Society for Industrial and Applied Mathematics.

Betts, J. T., Cramer, E. J., 1995. Application of Direct Transcription to Commercial Aircraft Trajectory Optimization. Journal of Guidance, Control and Dynamics 18 (1), 151-159.

Bonami, P., Olivares, A., Soler, M., Staffetti, E., sep 2013. Multiphase MixedInteger Optimal Control Approach to Aircraft Trajectory Optimization. Journal of Guidance, Control, and Dynamics 36 (5), 1267-1277.

Bronsvoort, J., 2014. Contributions to Trajectory Prediction Theory and its application to arrival management for Air Traffic Control. Ph.D. thesis, Universidad Politécnica de Madrid. 
Chaloulos, G., Crück, E., Lygeros, J., 2010. A simulation based study of subliminal control for air traffic management. Transportation Research Part C: Emerging Technologies 18 (6), 963-974.

Coppenbarger, R. A., Lanier, R., Sweet, D., Dorsky, S., 2004. Design and Development of the En Route Descent Advisor (EDA) for Conflict-Free Arrival Metering. In: Metering, Proceedings of the AIAA Guidance, Navigation, and Control Conference. pp. 1-19.

Coppenbarger, R. A., Mead, R., Sweet, D., 2007. Field evaluation of the tailored arrivals concept for datalink-enabled continuous descent approach. In: AIAA Aviation Technology, Integration and Operations Conference (ATIO). Vol. 46.

De Jong, P. M. A., 2014. Continuous Descent Operations using Energy Principles. Ph.D. thesis, TU Delft.

ERAT Consortium, 2012. Final Report. Tech. rep., Environmentally Responsible for Air Transport (ERAT) Project, Sixth Framework Programme, E.C.

Federal Aviation Administration, 2014. NextGen Priorities Joint Implementation Plan. Tech. rep., Federal Aviation Administration.

Franco, A., Rivas, D., 2014. Analysis of optimal aircraft cruise with fixed arrival time including wind effects. Aerospace Science and Technology 32 (1), 212222 .

Franco, A., Rivas, D., Valenzuela, A., jan 2010. Minimum-Fuel Cruise at Constant Altitude with Fixed Arrival Time. Journal of Guidance, Control, and Dynamics 33 (1), 280-285.

Gong, C., Sadovsky, A., sep 2010. A Final Approach Trajectory Model for Current Operations. In: 10th AIAA Aviation Technology, Integration, and Operations (ATIO) Conference. American Institute of Aeronautics and Astronautics, Reston, Virigina. 
Hartjes, S., Visser, H. G., Hebly, S. J., 2010. Optimisation of RNAV noise and emission abatement standard instrument departures. The Aeronautical Journal 114 (1162), 757-767.

Johnson, C. M., 2011. Analysis of Top of Descent (TOD) Uncertainty. In: Digital Avionics Systems Conference (DASC), 2011 IEEE/AIAA 30th. IEEE, pp. $1-10$.

Mondoloni, S., 2009. Use of Linear Aircraft Intent Response for Tactical Trajectory Based Operations. Eighth USA/Europe Air Traffic Management Research and Development Seminar (ATM2009).

Prats, X., 2010. Contributions to the optimisation of aircraft noise abatement procedures. Ph.D. thesis, Universitat Politècnica de Catalunya.

Prats, X., Bussink, F., Verhoeven, R., Marsman, A., sep 2015. Evaluation of in-flight trajectory optimisation with time constraints in a moving base flight simulator. In: Proceedings of the 34th Digital Avionics Systems Conference. IEEE/AIAA, Prague (Czech Republic).

Prats, X., Puig, V., Quevedo, J., jan 2011. A multi-objective optimization strategy for designing aircraft noise abatement procedures. Case study at Girona airport. Transportation Research Part D: Transport and Environment 16 (1), $31-41$.

Ruiz, S., Piera, M. a., Nosedal, J., Ranieri, A., 2014. Strategic de-confliction in the presence of a large number of $4 \mathrm{D}$ trajectories using a causal modeling approach. Transportation Research Part C: Emerging Technologies 39, 129147.

SESAR Consortium, 2012. European ATM Master Plan - Edition 2. Tech. Rep. October, Single European Sky ATM Research.

Soler, M., Kamgarpour, M., Lygeros, J., 2014a. A Numerical Framework and Benchmark Case study for Muti-modal Fuel Efficient Aircraft Conflict Avoid- 
ance. In: 6th International Conference on Research in Air Transportation (ICRAT).

Soler, M., Zou, B., Hansen, M., 2014b. Flight trajectory design in the presence of contrails: Application of a multiphase mixed-integer optimal control approach. Transportation Research Part C: Emerging Technologies 48, 172-194.

Thompson, T., Miller, B., Murphy, C., Augustine, S., White, T., Souihi, S., 2013. Environmental Impacts of Continuous-descent Operations in Paris and New York Regions. In: USA/Europe Air Traffic Management Research and Development Seminar.

Vilardaga, S., Duan, P., Prats, X., Uijt de Haag, M., 2014. Conflict Free Trajectory Optimisation with Target Tracking and Conformance Monitoring. In: 14th AIAA Aviation Technology, Integration, and Operations Conference, AIAA Aviation and Aeronautics Forum and Exposition 2014. Vol. 45701. pp. $1-12$.

Vilardaga, S., Prats, X., 2014. Conflict free trajectory optimisation for complex departure procedures. In: 6th International Conference on Research in Air Transportation (ICRAT). Istanbul.

Visser, H. G., Hartjes, S., 2014. Economic and environmental optimization of flight trajectories connecting a city-pair. Proceedings of the Institution of Mechanical Engineers, Part G: Journal of Aerospace Engineering 228 (6), 980-993.

Yang, L., Qi, J., Xiao, J., Yong, X., jun 2014. A literature review of UAV 3D path planning. In: Intelligent Control and Automation (WCICA), 2014 11th World Congress on. pp. 2376-2381. 